\title{
Spitzer IRS spectra of compact Galactic planetary nebulae: The link between dust, evolution, and metallicity
}

\author{
L. Stanghellini ${ }^{1}$, D. A. García-Hernández ${ }^{2,3}$, P. García-Lario ${ }^{4}$, \\ J. E. Davies ${ }^{5}$, R. A. Shaw ${ }^{1}$, E. Villaver ${ }^{6}$, A. Manchado ${ }^{2,3,7}$, \\ and J. V. Perea-Calderón ${ }^{8}$ \\ ${ }^{1}$ National Optical Astronomy Observatory Tucson, AZ 85719, USA \\ ${ }^{2}$ Instituto de Astrofísica de Canarias, vía Láctea s/n, La Laguna, E-38200 Tenerife, Spain \\ ${ }^{3}$ Departamento de Astrofísica, Universidad de La Laguna, E-38201 Tenerife, Spain \\ ${ }^{4}$ Herschel Science Centre, European Space Astronomy Centre, E-28080 Madrid, Spain \\ ${ }^{5}$ Spitzer Science Center, California Institute of Technology, Pasadena, CA 91125, USA \\ ${ }^{6}$ Departamento de Física Teórica C-XI, Univ. Autónoma de Madrid, E-28049 Madrid, Spain \\ ${ }^{7}$ Consejo Superior de Investigaciones Cientficas, E-28006 Madrid, Spain \\ ${ }^{8}$ European Space Astronomy Centre, INSA S. A., E28080, Madrid, Spain
}

\begin{abstract}
A sample of $\sim 150$ compact Galactic PNe has been observed with the Spitzer/IRS spectrograph to characterize their dust properties. These PNe are likely to be at the onset of the PN evolutionary phase, and are therefore ideal for probing dust evolution. The molecular emission features in these Galactic PN spectra are similar to those found in our Magellanic Cloud sample, except that we found a sizable fraction of PNe with mixed-chemistry dust which are not observed in the Clouds. We also found that the distribution among dust types depends strongly on the metallicity of the parent population, implying that the metallicity of the progenitors affects the evolution of a PN from its early stages.
\end{abstract}

Keywords. Planetary nebulae: general, dust, infrared: stars

\section{Motivation}

There are more than 2000 spectroscopically confirmed PNe in the Galaxy (Acker et al. 1992; Parker et al. 2006; Miszalski et al. 2008), and about 200 of them have not been spatially resolved in ground-based optical images. Thus, their detailed shapes, size, and central stars (CS) have not been studied. These compact PNe (with $\theta<4^{\prime \prime}$ ) are typically young, having left the AGB less than $\sim 2000 \mathrm{yr}$ ago if they are closer than $2 \mathrm{kpc}$ (Villaver et al. 2002). Compact PNe are essential to understand the onset of morphology and the early role of dust. Yet when studying Galactic PN populations (i.e., chemical gradients) compact $\mathrm{PNe}$ are generally excluded.

We thus embarked on a major, two-fold, NASA Great Observatories project including a Spitzer IRS spectroscopic survey of 157 compact $\left(\theta \leqslant 4^{\prime \prime}\right)$ Galactic PNe to study dust properties and gas phase elemental abundances. This study includes all such compact Galactic PNe not previously observed with the IRS. We also initiated an HST WFC3 image survey of a subset of these targets to study the onset of morphology and its relation to chemistry and dust, and the evolutionary state of the central stars of the sample (Shaw et al., in prep). The targets in our program are representative of the entire Galactic population in its distribution across the Galactic disk and in distance from the plane. While most Galactic bulge PNe have been observed with Spitzer by others (e.g., Perea-Calderon et al. 2009), we still found 30 bulge PNe in our sample, using the same selection criteria as Stanghellini \& Haywood (2010). 


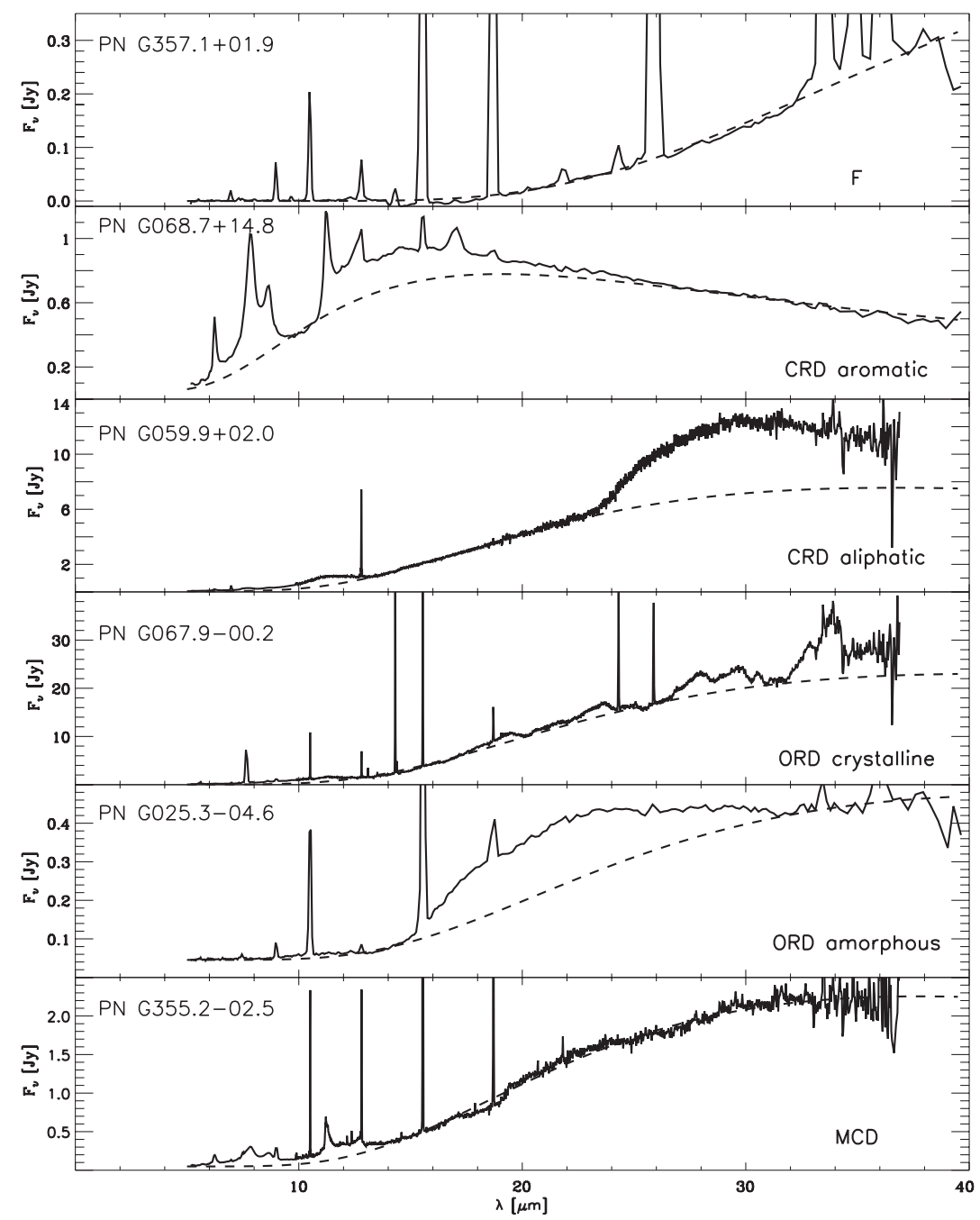

Figure 1. Spectral types encountered in our compact Galactic PN survey.

\section{The spectra}

All PN spectra show contributions from thermal dust continuum and emission lines from various atomic species. Molecular emission lines and bands are also commonly, but not always, present. These emission features in the spectra are those characteristic of carbon-rich dust, such as aromatic and aliphatic emission, and oxygen-rich dust, both crystalline silicates and amorphous types. We classify the spectra based on dust type, as illustrated in Figure 1, as F (featureless, except for thermal continuum and atomic emission), CRD (carbon-rich dust), ORD (oxygen-rich dust), and MCD (mixed-chemistry dust) if both CRD and ORD features are seen.

We found that about $20 \%$ of the sample has type F spectra, while the rest of the spectra are almost equally divided between the CRD, ORD, and MCD classes. This statistical result is very different from that of the Magellanic Cloud PNe (Stanghellini et al. 2007), where ORD are a minority and MCD are entirely absent. In Figure 2 we show the number of PNe for each dust class as a function of the metallicity of the parent population, expressed as $\mathrm{O} / \mathrm{H}$. There is continuity across populations, where the $\mathrm{F}$ and 


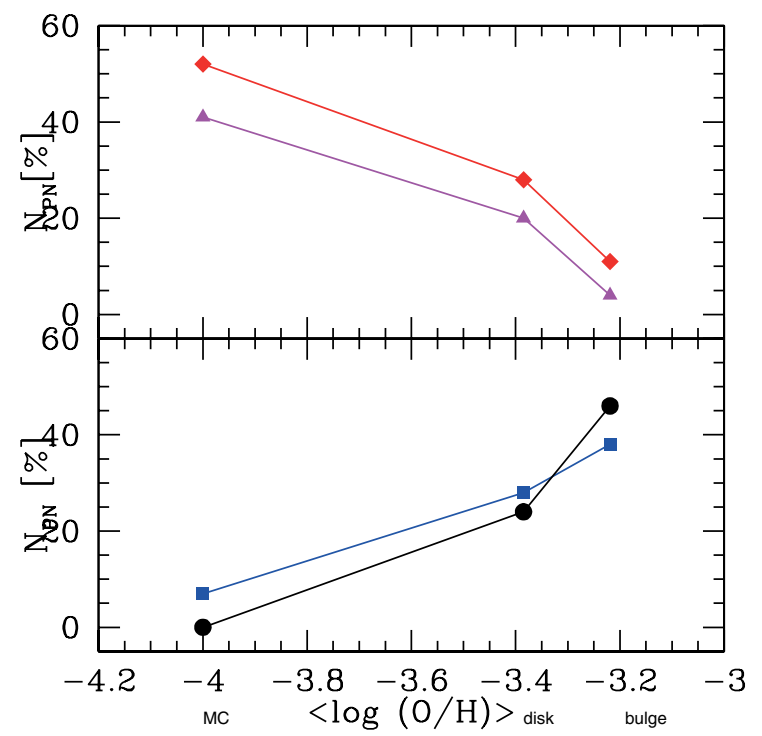

Figure 2. Galactic disk, bulge, and Magellanic Cloud PN fraction of dust types: F (triangles), CRD (diamonds), ORD (squares), and MCD (circles). Oxygen abundances from Stanghellini \& Haywood (2010), and Stanghellini (2003). Magellanic Cloud dust types include the Stanghellini et al. (2007) and Bernard-Salas et al. (2009) samples.

CRD classes are more populated with decreasing metallicity, and the opposite for ORD and MCD PNe.

There is also continuity between the bulge, the disk, and the Magellanic Clouds, in that there is a clear sequence in metallicity, where metal rich environments favor oxygenrich and mixed-chemistry dust, while low-metallicity environments favor the formation of carbon dust. Dust emission features (above the thermal continua) are more frequent in metal-rich environments. Within the PNe with solid state features, CRD is predominant in the Magellanic Clouds where crystalline silicates and other oxygen features are very rare, and mixed chemistry is not observed. Oxygen features, both crystalline and amorphous, are predominant in Galactic PNe. Galactic bulge PNe show majority of mixed-chemistry dust types.

\section{Discussion}

Dust class frequency is clearly linked with metallicity of the (progenitor) population. Since dust is closely related to the evolution of a PN (through pressure on dust grains at ejection, early and late evolution), $\mathrm{PNe}$ with different dust chemistry might evolve differently. As a consequence, overall PN populations in or within galaxies could be very different if their metallicity is different. To shed some light in these complicated issues we study the evolution of the different dust classes. In Figure 3 we plot the dust temperature of the sample PNe, as measured by continuum-fitting of the black-body like dust continua below the discrete dust features, against the nebular radii. Distances to the PNe are from Stanghellini \& Haywood (2010). $R_{\mathrm{PN}}$ is the independent evolutionary variable. Here we plot the pure Galactic disk population, excluding the bulge PNe. It is clear that CRD $\mathrm{PNe}$ form an evolutionary sequence, which means a narrow range of progenitor masses and metallicity. Since $T_{\text {dust }} \leqslant R_{\mathrm{PN}}^{0.4}$ during the evolution of a PN at constant luminosity, and even more steeply after the luminosity declines (Lenzuni et al. 1989), the CRD PNe 


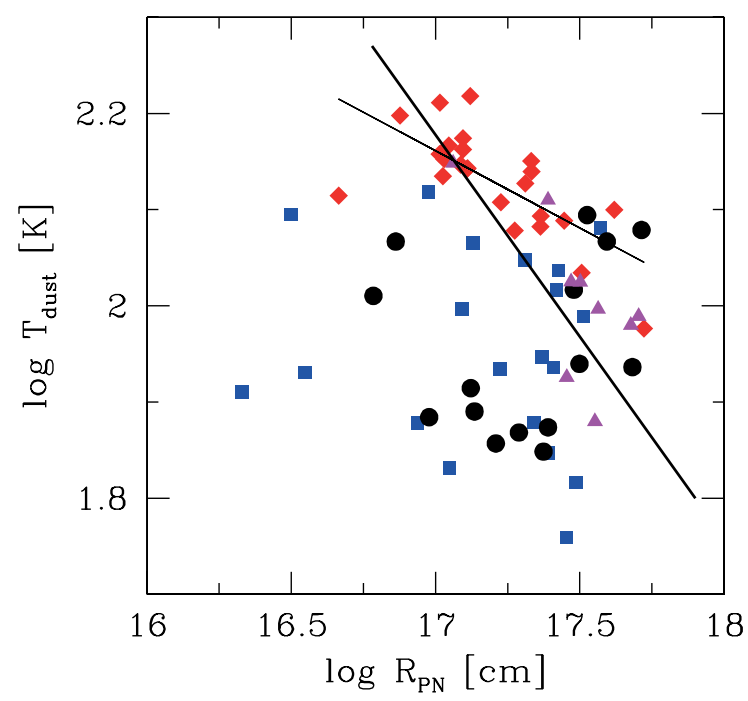

Figure 3. The decline in dust continuum temperature with nebular radius illustrates the evolution of dust grains in CRD PNe as a tight evolutionary sequence. Most of the F PN are found at large radii, as expected for the evolved dust features to become invisible. The thin line is a fit for the CRD sequence, while the thicker line is the relation for Galactic PNe if no grain evolution is expected. Symbols as in Fig. 2.

clearly show grain evolution (as well as nebular evolution) where the size of grains must decrease with time. More discussion on this plot and the grain evolution for the different dust types will be published elsewhere (Stanghellini et al. 2011, submitted).

\section{Future work}

The Spitzer IRS spectra discussed here have been analyzed for the presence of complex carbon-based molecules such as fullerenes and graphene precursors (García-Hernández et al. 2010, 2011) but a more comprehensive molecular analysis is currently being performed. Preliminary investigation on the WFC3/HST data show that there is a mild correlation between morphological types and dust class in Galactic PNe as was found very clearly in Magellanic Cloud PNe. A statistical analysis of the sample and the study of the grain evolution may be found in Stanghellini et al. (2011, submitted).

\section{References}

Acker, A., Marcout, J., Ochsenbein, F., Stenholm, B., Tylenda, R., \& Schohn, C. 1992, The Strasbourg-ESO Catalogue of Galactic Planetary Nebulae. Parts I, II. (Garching, Germany: European Southern Observatory)

Bernard-Salas, J., Peeters, E., Sloan, G. C:, et al. 2009, ApJ, 699, 1541

García-Hernández, D. A., Manchado, A., García-Lario, P., et al. 2010, ApJ, 724, L39

García-Hernández, D. A., Iglesias-Groth, S., Acosta-Pulido, J. A., et al. 2011, ApJ, 737, L30

Lenzuni, P., Natta, A., \& Panagia, N. 1989, ApJ, 345, 306

Miszalski, B., Parker, Q. A., Acker, A., et al. 2008, MNRAS, 384, 525

Parker, Q. A., Acker, A., Frew, D. J., et al. 2006, MNRAS, 373, 79

Perea-Calderón, J. V., García-Hernández, D. A., García-Lario, P., et al. 2009, A 6 A, 495, L5

Stanghellini, L., García-Lario, P., García-Hernández, D. A., et al. 2007, ApJ, 671, 1669

Stanghellini, L. \& Haywood, M. 2010, ApJ, 714, 1096

Villaver, E., Manchado, A., \& García-Segura, G. 2002, ApJ, 581, 1204 